\title{
OPTICAL AND PHOTOEMISSION PROPERTIES OF GRAPHITE GRAINS
}

\author{
R. F. WILLIS, B. FEUERBACHER, and B. FITTON \\ Surface Physics Division, European Space Research Organisation, Noordwijk, Holland
}

\begin{abstract}
The optical properties and collective excitations are discussed for graphite and vitreous carbon. The bulk resonance energy for the $\pi$-plasmon is seen to be shifted by $1.4 \mathrm{eV}$ from the crystalline to the amorphous case, indicating that the degree of structural disorder is an important parameter in the optical properties of carbon grains. Data are presented on work function, photoelectric yield, and photoelectron energy distribution for both graphite and vitreous carbon. Under solar irradiation, photoelectrons from graphite are emitted with an average energy of about $1 \mathrm{eV}(12000 \mathrm{~K})$.
\end{abstract}

Multi-component models of interstellar grains (Bless and Savage, 1972; Hoyle and Wickramasinghe, 1962; Wickramasinghe and Nandy, 1970; Gilra, 1971) have been widely used to match the observed absorption and polarisation features of interstellar extinction. Observations in the far UV (Stecher, 1965; Bless and Savage, 1972) have revealed a pronounced structure in the extinction curve, located at $2175 \AA$ wavelength. This structure has been attributed to absorption by small graphite particles (Stecher and Donn, 1965; Gilra, 1971), which are assumed to be one of the major constituents of the interstellar matter. Until recently the basic optical properties of bulk graphite were not completely understood, which led to difficulties in predicting the optical response of small graphite particles. In this paper, results of experimental studies on the optical and photoemission properties of graphite and of vitreous carbon, which is a highly disordered or glassy form of carbon, are presented. These experiments were performed in order to provide data to support calculations of the extinction due to graphite grains and the grain charging due to photoelectric emission.

\section{The Optical Properties of Graphite Grains}

The optical properties of graphite for light polarized with the electric field vector normal to the $c$-axis $(E \perp c)$ have been measured over a wide energy range by Taft and Philipp (1965). Optical measurements for $E / / c$ are available only up to $10 \mathrm{eV}$ $(1240 \AA)$. In order to determine the optical constants for higher energies, Tosatti and Bassani (1970) applied a Kramers-Kronig analysis to the electron energy-loss data of Zeppenfeld (1968) and derived a strong $E / / c$ absorption peak near $11 \mathrm{eV}(1130 \AA)$. However recent experimental studies of the energy band structure of graphite (Willis et al., 1971) do not endorse this assignment of an $E / / c$ electronic transition at $11 \mathrm{eV}$. The lowest transition for $E / / c$ that could give rise to optical absorption in this direction of polarization was found to lie at $14.5 \mathrm{eV}(855 \AA)$. This result is in agreement with a recent band-structure calculation by Painter and Ellis (1970), and with optical measurements performed with a synchrotron light source (Klucker and Skibowski, 
unpublished). From these results it is concluded that crystalline graphite is essentially transparent for light polarized with $E / / c$ at least up to the interstellar cutoff at $13.6 \mathrm{eV}$.

Stecher and Donn (1965) have calculated the extinction due to small graphite particles using Mie's theory and the optical constants of Taft and Philipp (1965). They found an absorption peak near $2200 \AA$ in close agreement with the observed bump in the extinction curve. This structure has been considered as due to an electronic interband transition of the $\pi$-electrons in graphite (Stecher, 1969). However accurate thermoreflectance measurements (Anderegg et al., 1971) have located the $\pi-\pi$ transition at $2580 \AA$ rather than at $2200 \AA$. so that additional effects must be contributing to the observed absorption features. As pointed out by van de Hulst (1957), the role of collective electron oscillations (plasmons) has to be considered. In fact, collective processes may become the dominant absorption feature in cases when the size of the particles becomes small compared to the wavelength of the light, as shown for example by Genzel and Martin (1973). While of course the plasmon treatment does not give results different from the Mie theory, Gilra (1973) has shown that a study of the peak position of the plasma resonance allows an easier means of drawing conclusions on size, shape, and coatings of the grains, at least in the small particle limit. Using dielectric constants of graphite as given by Taft and Philipp (1965) he concluded that the particles should be very small ( $<150 \AA$ ), spherical, and free of any coating.

There is some uncertainty concerning the degree of crystallographic order in interstellar grains. While it is not expected that the grain will be free of defects, mainly because of the bombardement by energetic particles and cosmic rays (Watson, 1972), it is also unlikely that the grains are composed of highly disordered vitreous carbon. Optical data on both these limiting cases of structure are available. Measurements on vitreous carbon have been published by Taft and Philipp (1965) and extended recently by Williams and Arakawa (1973). Electronic interband transition features are found at the same energies as with graphite for $E \perp c$ though somewhat broadened. Marked differences however are observed for the plasma resonance frequencies. The bulk plasmon energies for collective excitation of the $\pi$-electrons and of the $\pi$ - and $\sigma$-electrons, observed at $7 \mathrm{eV}(1770 \AA)$ and $25 \mathrm{eV}$ for graphite, are found to be shifted to $5.6 \mathrm{eV}(2210 \AA)$ and $20.4 \mathrm{eV}$ for vitreous carbon. This shift in plasma resonance frequency depends on the electron density in the amorphous material. From this point of view, some of the stringent limitations imposed on size, shape, and coatings deduced from the plasmon model by Gilra (1973) have to be treated with care. Given various degrees of micro-structural disorder one can arrive at a variety of possible sizes and shapes for interstellar carbon grains which could fit the observed extinction curve in the region around $2200 \AA$.

\section{Photoemission and Charging of Graphite Grains}

The problem of photoemission from interstellar grains has received little attention to date, primarily due to a lack of information on the galactic UV radiation field and the photoemission properties of grain materials. Previous calculations of the charge on 
grain surfaces indicated negatively charged particles as a result of the equilibrium between the sticking of electrons and positive ions in the ambient plasma (Spitzer, 1948, Wickramasinghe, 1967). However recent investigations (Watson, 1974), in which an estimate of the photoelectron yield has been made, indicate positively charged grains.

Attempts to calculate the electrostatic surface charge require information concerning the photoelectric threshold and quantum yield of the grain material, the energy distribution of the photoemitted electrons, and the ambient charged particle flux density, sticking probability, and kinetic temperature.

Figure 1 shows the measurement of the work function (photoelectric threshold) of graphite, using the Fowler method. A straight line extrapolation of the square root of the yield plotted against photon energy gives a work function value of $4.7 \mathrm{eV}(2640 \AA)$ for graphite. A work function of $4.75 \mathrm{eV}$ was obtained for the vitreous carbon sample by the same method. The value of the work function is a particularly important parameter for the determination of the grain potential in those regions where the photon flux is large compared to the incident particle flux onto the grain.

The photoelectric yield in terms of emitted electrons per incoming photon is given in Figure 2 as a function of photon energy for both graphite and vitreous carbon. The yield of graphite is found to be exceptionally small compared to other materials (Feuerbacher and Fitton, 1972), reaching a maximum value of only 0.03 at $15 \mathrm{eV}$. In contrast, metals may have a value ten times this in the same energy range, whilst the yield for insulators can approach unity. In the range up to $13.6 \mathrm{eV}$ the photoelectric yield of graphite does not exceed $2 \%$, and in the 1000-2000 $\AA$ region the average value is about $10^{-4}$. Slightly higher values are found for vitreous carbon. The inset shows the observed reflectance in the same energy range. It can be seen that the

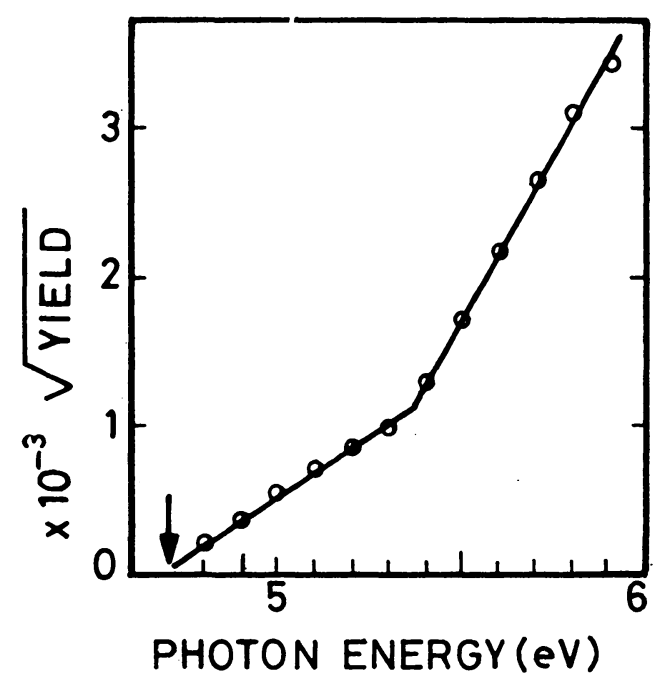

Fig. 1. Determination of the work function of graphite using Fowlers method. 


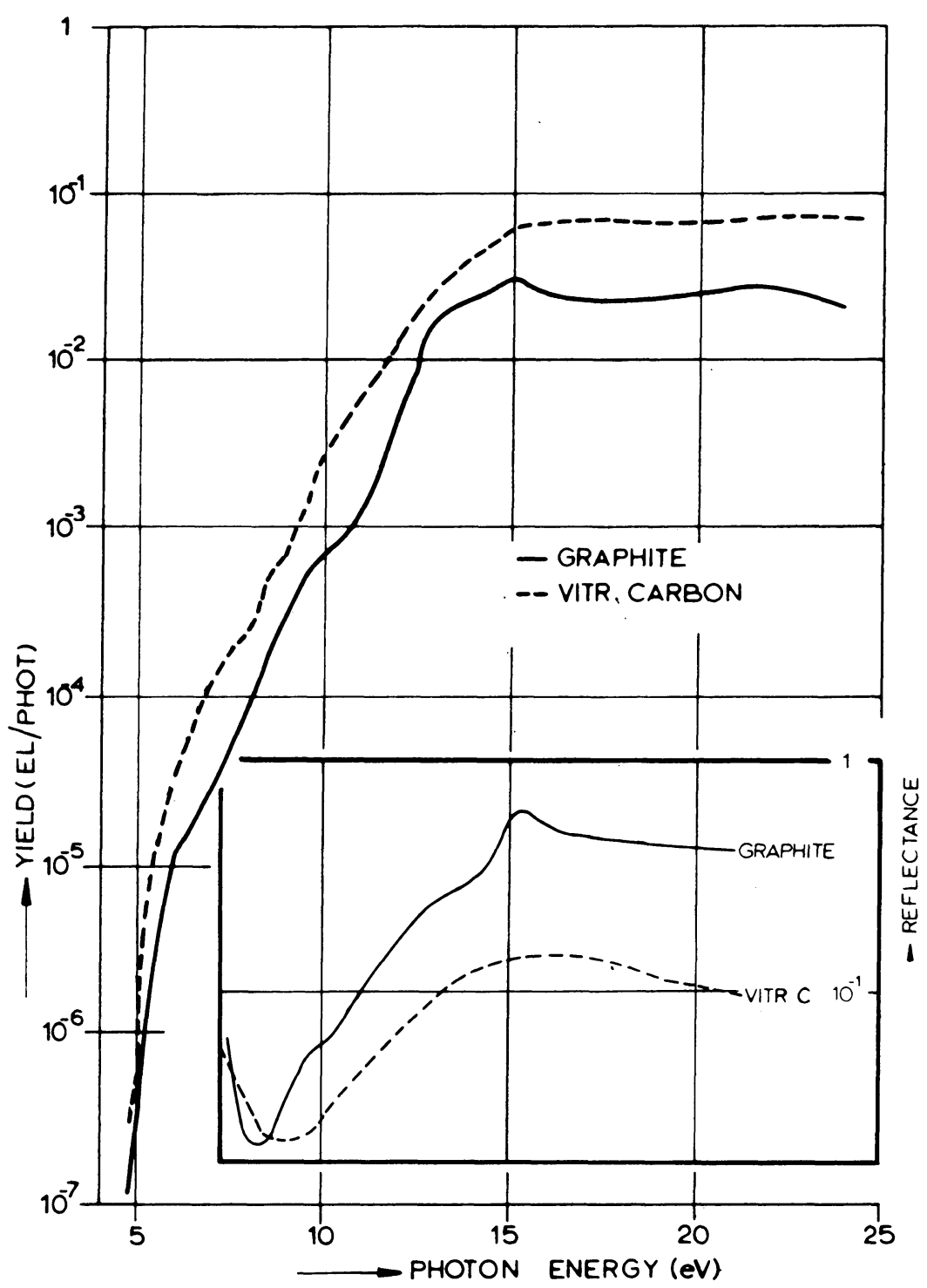

Fig. 2. Photoelectric yield per incident photon for graphite and vitreous carbon. The inset gives the reflectance in the same photon energy scale.

higher yield of vitreous carbon may be partly accounted for by the lower reflectivity of this material.

A set of photoelectron energy distribution curves for graphite is shown in Figure 3. Each curve has been scanned at a photon energy that may be read from the right hand scale. The number of electrons emitted in an energy interval is plotted as a function of electron energy. The curves are normalized such that the integral under each spectrum equals unity. All spectra are seen to peak at relatively low electron 


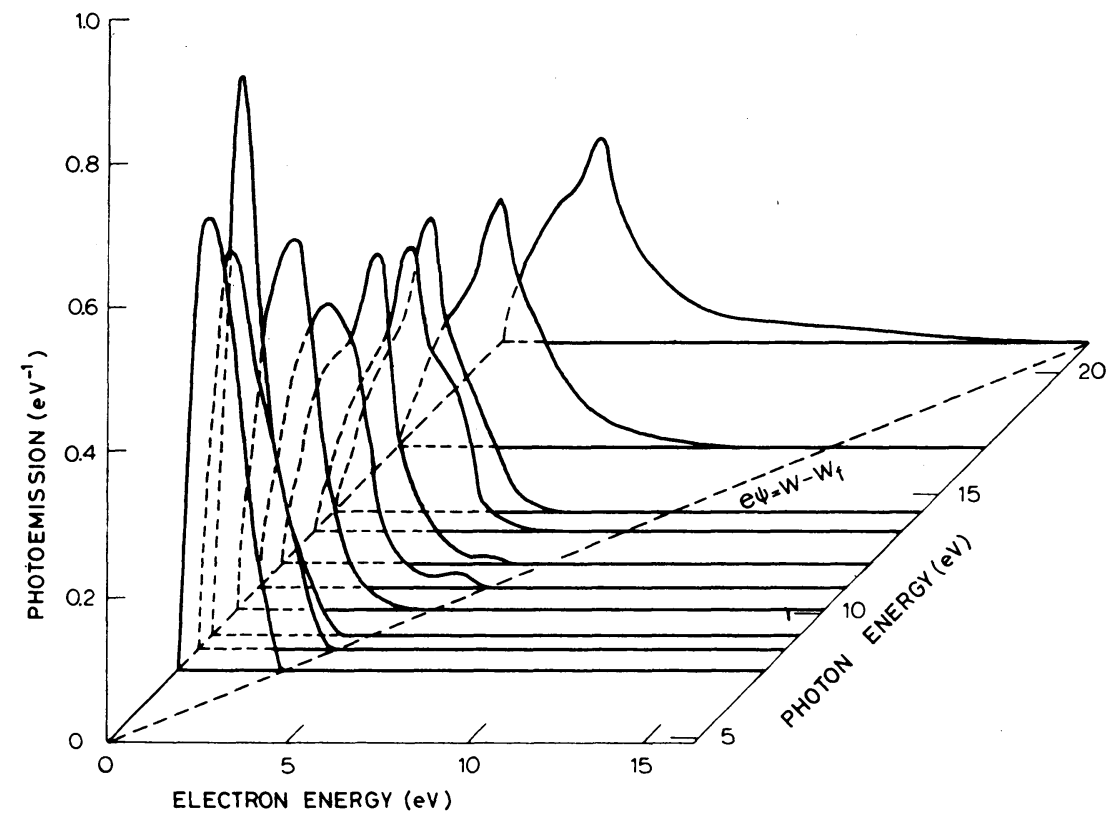

Fig. 3. Electron energy distribution of photoelectrons emitted from graphite for various photon energies as given on the right hand scale. All spectra are normalized to unity area under the curves.

energies. Spectra taken with photon energies above $11 \mathrm{eV}$ show a pronounced peak at $2.8 \mathrm{eV}$. It is important to note that very few electrons are emitted with energies close to the highest possible energy, which is the photon energy minus the work function, given as the dashed straight line in Figure 3.

Providing the spectrum of the light incident on the particle is known, the curves of Figure 3 may be integrated to obtain the total energy distribution of the electrons

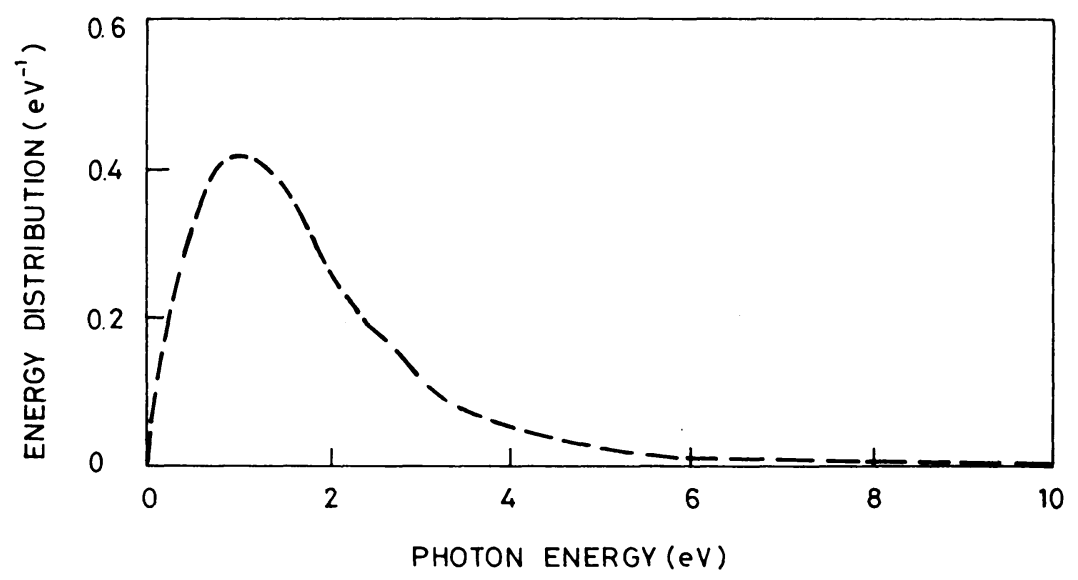

Fig. 4. Total energy distribution of photoelectrons emitted from graphite under solar irradiation at $1 \mathrm{AU}$, normalized to unity area. 
emitted under this illumination. This has been done in Figure 4, using the solar spectrum as the source of illumination. Again the curve is normalized such that the area under it equals unity. The curves are calculated (Grard, unpublished) for the case of a particle small compared to the Debye length of the surrounding plasma. These and other calculations have been performed in the course of investigations concerning the charging of satellites in space (Grard, unpublished) or the lunar surface (Feuerbacher et al., 1973). In Figure 4 it can be seen that the peak electron energy is approximately $1 \mathrm{eV}$. The strong structure at $2.8 \mathrm{eV}$ in the energy distributions of Figure 3 is seen only as a slight hump at this energy for the case of solar irradiation. The observed low average electron energy, corresponding to an electron temperature of about $12000 \mathrm{~K}$, is obtained due to the strong increase of the intensity of the solar spectrum, which is essentially a $6000 \mathrm{~K}$ blackbody spectrum, for wavelengths below $1400 \AA$, thus emphasizing the low electron-energy region.

It has been shown (Grard, unpublished) that for most materials the photoelectron energy distribution curve may be approximated by a Maxwellian distribution, at least for the particular case of solar irradiation. Using this approximation an analytical expression has been derived (Grard and Tunaley, 1971) to calculate the potential of a body or of particles in space. Data for the case of interstellar grains has been published (Feuerbacher et al., 1973).

In summary, the optical properties of small grains are likely to be dominated by collective electron effects and modified somewhat by structural disorder. The photoemission from graphite grains will be abnormally low compared to that of most materials with a maximum in the emission energy of about $1 \mathrm{eV}$ for the particular case of solar irradiation.

\section{Acknowledgements}

We thank Dr R. J. L. Grard and Dr D. P. Gilra for helpful discussions, Dr E. T. Arakawa for providing the optical data on vitreous carbon prior to publication, and Dr E. A. Trendelenburg for his encouragement with this work.

\section{References}

Anderegg, M., Feuerbacher, B., and Fitton, B.: 1971, Phys. Rev. Letters 26, 760.

Bless, R. C. and Savage, B. D.: 1972, Astrophys. J. 171, 293.

Carter, J. G., Huebner, R. H., Hamm, R. N., and Birkhoff, R. D.: 1965, Phys. Rev. 137, A639.

Feuerbacher, B. and Fitton, B.: 1972, J. Appl. Phys. 43, 1563.

Feuerbacher, B., Anderegg, M., Fitton, B., Laude, L. D., Willis, R. F., and Grard, R. J. L.: 1972 , Proc. 3rd Lunar Sci. Conf. Geochim. Cosmochim. Acta, Suppl. 3, 3, 2655.

Feuerbacher, B., Willis, R. F., and Fitton, B., 1973, Astrophys. J. 181, 101.

Genzel, L. and Martin, T. P.: 1973, Surface Sci. 34, 33.

Gilra, D. P.: 1971, Nature 229, 237.

Gilra, D. P.: 1971, this volume, p. 517.

Grard, R. J. L. and Tunaley, J. K. E.: 1971, J. Geophys. Res. 76, 2498.

Grard, R. J. L.: ESTEC IWP 663, European Space Research Organization, Noordwijk, Holland, unpublished. 
Greenaway, D. L., Harbeke, G., Bassani, F., and Tosatti, E.: 1969, Phys. Rev. 178, 1340.

Hoyle, F. and Wickramasinghe, N. C.: 1962, Monthly Notices Roy. Astron. Soc. 124, 417.

Klucker, R. and Skibowski, M.: DESY, Hamburg, Germany, unpublished.

Painter, G. S. and Ellis, D. E.: 1970, Phys. Rev. B1, 4747.

Spitzer, L.: 1948, Astrophys. J. 107, 6.

Stecher, T. P.: 1965, Astrophys. J. 142, 1683.

Stecher, T. P.: 1969, Astrophys. J. 157, L125.

Stecher, T. P. and Donn, B.: 1965, Astrophys. J. 142, 1681.

Taft, E. A. and Philipp, H. R.: 1965, Phys. Rev. 138, A197.

Tosatti, E. and Bassani, F.: 1970, Nuovo Cimento 65, 161.

Van de Hulst, H. C.: 1957, Light Scattering by Small Particles', John Wiley and Sons, New York.

Watson, W. D.: 1972, Astrophys. J. 176, 103 and 271.

Watson, W. D.: 1973, this volume, p. 335.

Williams, M. W. and Arakawa, E. T.: 1973, to be published.

Willis, R. F., Feuerbacher, B., and Fitton, B.: 1971, Phys. Rev. B4, 2941.

Wickramasinghe, N. C.: 1967, Interstellar Grains, Chapman and Hall, London.

Wickramasinghe, N. C. and Nandy, K.: 1970, Nature 227, 51.

Zeppenfeld, K.: 1968, Z. Physik 211, 391. 